\title{
Comparison of autofluorescence imaging bronchoscopy and white light bronchoscopy for detection of lung cancers and precancerous lesions
}

\author{
This article was published in the following Dove Press journal: \\ Patient Preference and Adherence \\ 6 July 2013 \\ Number of times this article has been viewed
}

\section{Yan Wang ${ }^{1, *}$ \\ Qing Wang ${ }^{2, *}$ \\ Jing Feng 1,3 \\ $\mathrm{QiWu}$}

'Respiratory Department of Tianjin Medical University General Hospital, Tianjin, People's Republic of China; ${ }^{2}$ Respiratory Department of the First People's Hospital of Kunming, Yunnan, People's Republic of China; ${ }^{3}$ Division of Pulmonary and Critical Care Medicine, Duke University Medical Center, Durham, NC, USA; ${ }^{4}$ Respiratory Department of Tianjin Haihe Hospital, Tianjin, People's Republic of China

*These two authors contributed equally to this work
Correspondence: Jing Feng Respiratory Department of Tianjin Medical University General Hospital, Tianjin 300052, People's Republic of China

Tel + I39 2050025 I

Fax $+86226036 \quad 632$

Email jing.feng2@duke.edu

Qi Wu

Respiratory Department of Tianjin Haihe Hospital, Tianjin 300350,

People's Republic of China

Tel +86 I 3920420866

Fax +86 $226036 \quad 632$

Emailwq572004@I63.com
Background: The purpose of this paper was to compare the sensitivity, specificity, and overall diagnostic performance of autofluorescence imaging bronchoscopy (AFI) versus white light bronchoscopy (WLB) in the detection of lung cancers and precancerous lesions by meta-analysis. Methods: We performed a literature search using the PubMed and EMBASE databases to identify studies published between March 1991 and March 2012. Article selection, quality assessment, and data extraction were then performed. The pooled sensitivity, specificity, diagnostic odds ratio, and area under the curve of the summary receiver operating characteristic for AFI versus WLB were calculated using Stata version 12.0 software.

Results: Six studies were included in the meta-analysis. The pooled sensitivity of AFI and WLB was 0.89 (95\% confidence interval [CI] 0.81-0.94) and 0.67 (95\% CI 0.46-0.83) and the pooled specificity of AFI and WLB was 0.64 (95\% CI 0.37-0.84) and 0.84 (95\% CI 0.74-0.91), respectively. The diagnostic odds ratio for AFI and WLB was 14.5 (95\% CI 3.76-55.63) and 10.9 (95\% CI 3.12-38.21), and the area under the curve for AFI and WLB was 0.89 (95\% CI 0.86-0.92) and 0.85 (95\% CI 0.81-0.88), respectively. The pooled positive and negative likelihood ratios were 2.5 (95\% CI 1.21-4.97) and 0.17 (95\% CI 0.08-0.36) for AFI, and the corresponding values for WLB were 4.3 (95\% CI 2.13-8.52) and 0.39 (95\% CI 0.21-0.73). The pooled positive likelihood ratio for AFI and WLB was not higher than 10, and the pooled negative likelihood ratio for AFI and WLB was not lower than 0.1 .

Conclusion: The sensitivity of AFI is higher than that of WLB, while the specificity of AFI is lower than that of WLB. The overall diagnostic performance of AFI is slightly better than that of WLB in detecting lung cancers and precancerous lesions. AFI should find its place in routine bronchoscopic examination and may improve the diagnostic outcome on endoscopy.

Keywords: autofluorescence imaging bronchoscopy, autofluorescence bronchoscopy, light bronchoscopy, lung cancers, precancerous lesions

\section{Introduction}

Recent epidemiological research shows that lung cancer is the most common cause of cancer-related death, and there is no doubt that early detection followed by prompt treatment can improve the outcome for patients with lung cancer and afford a better chance of cure. ${ }^{1}$ In patients with invasive forms of the disease, earlier detection of synchronous lesions may lead to significant changes in the clinical management protocol., ${ }^{2,3}$

There are several types of lung cancer, including small and nonsmall cell lung cancer. These cancers may arise from the peripheral (bronchioles and alveoli) or 
central (the major bronchi, central airways) structures of the lung. Adenocarcinoma and large cell carcinoma usually arise peripherally, whereas squamous cell carcinoma and small cell lung cancer usually arise centrally. Different approaches are required to detect cancer in different compartments of the lung.

Bronchoscopy is a desirable technology for detecting lung cancers arising from the central airways. However, even when performed by experienced bronchoscopists, it is still a challenge to detect early lung cancers and precancerous lesions using conventional white light bronchoscopy (WLB). It has been reported that only $29 \%$ of carcinoma in situ and $69 \%$ of microinvasive tumors are identified endoscopically on WLB, even by experienced bronchoscopists. ${ }^{4}$ Autofluorescence bronchoscopy is an advanced technology that exploits the autofluorescent nature of the bronchial mucosa to detect tiny and subtle superficial lesions. The difference in superficial autofluorescence intensity between normal tissue and tumor tissue was reported as far back as $1965,{ }^{5}$ and in the last two or three decades, autofluorescence bronchoscopy has been used to detect early-stage carcinomatous lesions through endoscopy workstations. ${ }^{6,7}$ Thus far, several types of medical autofluorescence bronchoscopy systems have been designed and developed, including the LIFE (Xillix Technologies, Richmond, Canada), D-Light (Karl Storz, Tuttlingen, Germany), and SAFE (Pentax, Tokyo, Japan) systems. In comparison with conventional WLB, these autofluorescence bronchoscopy systems have much higher sensitivity, which is interesting and exciting, but at the same time, the specificity is decreased, ${ }^{8-13}$ which is obviously a significant drawback.

Autofluorescence imaging bronchoscopy (AFI, Olympus Optical Corporation, Tokyo, Japan) is a newly developed autofluorescence bronchoscopy system. Major indications for AFI include: detection of early-stage lung cancers and precancerous lesions in the large central airways in high-risk patients; detecting subtle synchronous malignancy preoperatively in patients with lung cancer; evaluation of extension of lung cancer or evaluation of margins in endoscopically visible tumors; selection of the optimal surgical procedure in patients with early-stage lung cancer; and bronchoscopic surveillance during follow-up of patients after curative surgery for nonsmall cell lung cancer. AFI has been shown to be a far more sensitive method for detecting superficial lung cancers and precancerous lesions compared with WLB (see Figure 1). ${ }^{14-16}$ However, when AFI is compared with WLB for specificity, the literature yields conflicting results. ${ }^{14-19}$

The AFI system consists of three parts, ie, an autofluorescence video bronchoscope (BF-F260), a video processor
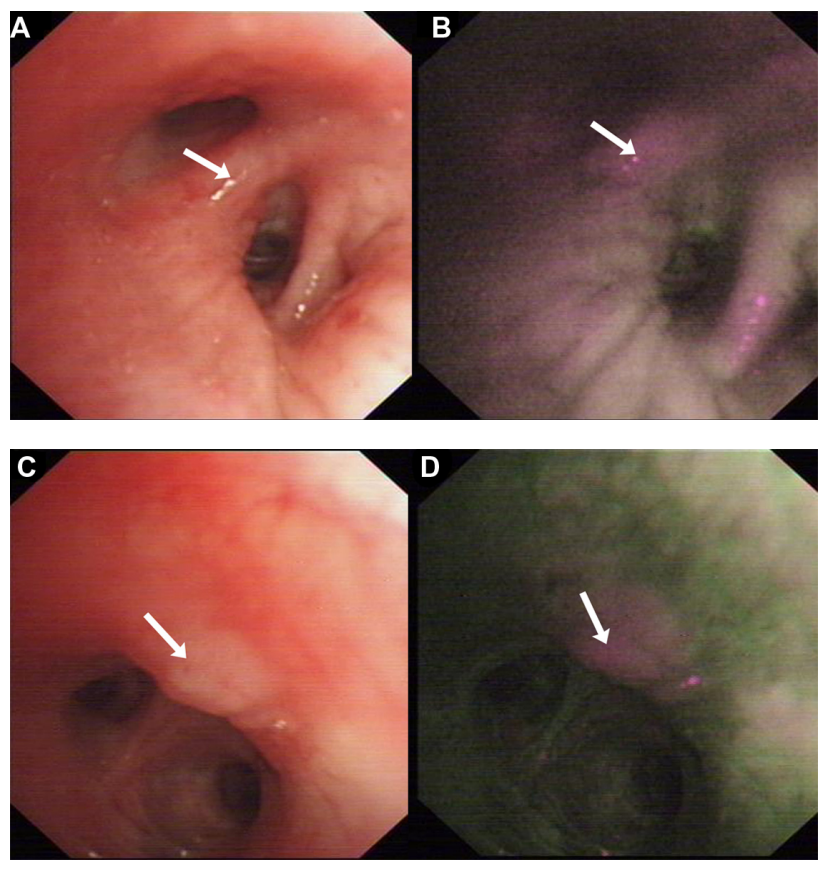

Figure I (A and B) WLB and AFI findings of adenocarcinoma at the bifurcation of the right middle lobe and lower lobe. (A) WLB image shows nonspecific swelling or thickening, may be treated as normal, and is not generally biopsied. (B) AFI image shows significant magenta color (white arrows indicate bifurcation of the right middle lobe and lower lobe). (C and D) WLB and AFI findings of small cell lung cancer on the wall of the left upper lobe. (C) Nonspecific protruding nodule can be found at the wall of the left upper lobe, may be treated as normal and is not generally biopsied. (D) AFI image shows significant magenta color (white arrows indicate a protruding nodule on the wall of the left upper lobe).

Abbreviations: AFI, autofluorescence imaging bronchoscopy; WLB, white light bronchoscopy.

unit (Evis Lucera Spectrum, CV-260SL), and a xenon light source. The autofluorescence video bronchoscopic image is presented on a 19-inch LCD monitor (OEV-191). The AFI system incorporates autofluorescence light (blue excitation light produces an autofluorescence signal at 490-700 nm, which is treated as green in the video processor), $\mathrm{G}^{\prime}$ reflected light (green, treated as red in the video processor) and $\mathrm{R}^{\prime}$ reflected light (red, treated as blue in the video processor). These three signals are synthesized by the video processor and a composite image is displayed on the LCD monitor. ${ }^{20}$ The onscreen image shows normal bronchial mucosa as green, ie, the color of general autofluorescence light. Because hemoglobin absorbs a large volume of autofluorescence light and $\mathrm{G}^{\prime}$ reflected light and little $\mathrm{R}^{\prime}$ reflected light, areas containing more hemoglobin show decreased autofluorescence (green) and $\mathrm{G}^{\prime}$ reflected light (red) but not $\mathrm{R}^{\prime}$ reflected light (blue). Therefore, areas containing more hemoglobin (local bleeding or bronchitis) produce a blue or green-blue color on AFI imaging. ${ }^{14}$ Loss of normal green autofluorescence can be caused by increased thickness of the epithelial layer and accumulation of cancerous material in superficial local 
tissue, ${ }^{21}$ which are the characteristics of early lung cancers and precancerous lesions. As a result, early lung cancers and precancerous lesions produce a magenta color, ie, a combination of red ( $\mathrm{G}^{\prime}$ reflected light) and blue ( $\mathrm{R}^{\prime}$ reflected light), in the AFI image (see Figure 1).

Overall, autofluorescence bronchoscopy has much higher sensitivity and decreased specificity in comparison with WLB. ${ }^{22}$ However, some reports ${ }^{14,17}$ have demonstrated that AFI, a newly developed specialized type of autofluorescence bronchoscopy, has relatively superior specificity compared with WLB, while others ${ }^{15,16}$ have shown similar or even lower specificity. To determine if AFI can be used in routine practice to improve the outcome of endoscopic diagnosis of lung cancers and precancerous lesions, we performed a meta-analysis comparing the sensitivity, specificity, and overall diagnostic performance between AFI and WLB, and to our knowledge, ours is the first exclusive meta-analysis of AFI.

\section{Materials and methods \\ Data source and search strategy}

We did a literature search using the PubMed and EMBASE databases to identify studies comparing the diagnostic results of AFI and WLB with regard to detecting lung cancers or precancerous lesions. We used the search terms "autofluorescence bronchoscopy", "autofluorescence imaging", and "AFI". Because autofluorescence bronchoscopy was first introduced in 1991, ${ }^{23,24}$ the literature published between March 1991 and March 2012 was examined. We then compared sources to exclude duplicate articles, and the search was further augmented by scanning references of identified articles or reviews for additional relevant papers.

\section{Eligibility criteria}

Papers were included if they used an AFI system and compared it with a WLB system, used histologic criteria as the golden standard when diagnosing lung cancers and precancerous lesions, and provided sufficient data (including true positives, false positives, false negatives, true negatives) to assess the diagnostic yield of AFI. When multiple publications involving the same study population were identified or study populations overlapped, only the larger study was included, unless the reported outcomes were mutually exclusive.

\section{Data extraction}

Data were extracted independently by two of the authors (YW, QW) and the final decision regarding inclusion was made by consensus. Data extracted from eligible studies included authors, study year, mean age and number of study participants, number of biopsies performed, biopsy strategy, and positive histologic standard used. True positive, false positive, false negative, and true negative numbers of AFI and WLB were extracted from the studies and two-dimensional tables were constructed.

\section{Assessment of quality}

The quality of each included study was assessed using the Quality Assessment of Diagnostic Accuracy Studies (QUADAS) tool. ${ }^{25}$ This tool is structured as a list of 14 questions which should be answered with, "yes", "no", or "unclear".

\section{Data synthesis and statistical analysis}

Stata version 12.0 (Stata Corporation, College Station, TX, USA) was used for all statistical analyses, in particular the Midas commands. We evaluate publication bias and heterogeneity, pooled sensitivity, specificity, and positive and negative likelihood ratios, and evaluated two global measures of accuracy of the tests, ie, the diagnostic odds ratio and summary receiver operating characteristic.

Publication bias is defined as the tendency on the part of investigators to submit and/or editors and reviewers to accept manuscripts based on the direction or strength of the study findings. This definition concentrates on the fact that the most positive and strongest studies are likely to be published, while negative results are less likely to be published in the journals, so publication bias is ubiquitous. Publication bias is a common problem for meta-analyses and cannot be avoided completely. Publication bias is generally described with funnel plots, and a symmetrical funnel plot indicates that publication bias does not exist. Linear regression as a test for funnel plot asymmetry was performed in our metaanalysis.

Heterogeneity can be expected in the results of studies of diagnostic test accuracy. ${ }^{26}$ Heterogeneity of results between studies is generally assessed graphically using forest plots and statistically by the quantity $I^{2}$ that describes the percentage of total variation across studies which is attributable to heterogeneity rather than chance. A value of $0 \%$ indicates no observed heterogeneity, and values greater than $50 \%$ are considered to indicate substantial heterogeneity. ${ }^{27}$

Sensitivity is defined as the number of true positives divided by the sum of true positives and false negatives. Specificity is defined as the number of true negatives divided by the sum of true negatives and false positives. The positive likelihood ratio is sensitivity divided by 1 - specificity, and indicates how much the odds of the disease increase when a 
test is positive. The likelihood ratio can be used to assess the impact of a positive test result on diagnosis for an individual. When the likelihood ratio is higher than 10 , we can make an almost definite diagnosis of a certain disease. The negative likelihood ratio is 1 - sensitivity divided by specificity, and indicates how much the odds of the disease decrease when a test is negative. When the likelihood ratio is $<0.1$, we can almost eliminate a certain disease. The diagnostic odds ratio of a test is the ratio of the positive likelihood divided by the negative likelihood ratio. The diagnostic odds ratio can be put forward as a useful single index of test performance indicating the strength of the association between test results and disease ${ }^{28}$ it is difficult to interpret clinically, but useful from the statistical point of view for evaluation of overall test accuracy in meta-analysis. ${ }^{28-30}$ The summary receiver operating characteristic is also a global tool for measuring the accuracy of tests. The results are described as the area under the curve (AUC) and the Q-point (point on curve where sensitivity equals specificity) obtained from the summary receiver operating characteristic curve. ${ }^{31} \mathrm{~A}$ bivariate binomial mixed model was selected to calculate the pooled sensitivity, specificity, positive likelihood ratio, negative likelihood ratio, and diagnostic odds ratio on a per lesion basis, and takes into account variability within and between studies and the dependency between sensitivity and specificity.

\section{Results \\ Study selection}

As seen in Figure 2, 2339 articles were identified by searching the PubMed and EMBASE electronic databases. Of these, 158 were retained for abstract review and then 21 for full text review. A further six full texts were identified by searching the references of these articles. After full text review, six studies were included in the meta-analysis. To avoid language bias, we did not exclude articles not reported in English. However, all the six articles finally included in our study were published in English.

\section{Quality of included studies}

The QUADAS tool results are shown in Table 1. Generally speaking, the studies included were ideal, in that they all fulfilled at least 12 of the 14 methodological criteria.

\section{Characteristics of included studies}

The characteristics of each study are listed in Table 2. A total of 1170 biopsy specimens from 409 patients examined with the AFI system and WLB system were included in the analysis. In all of the six articles, each sample was imaged

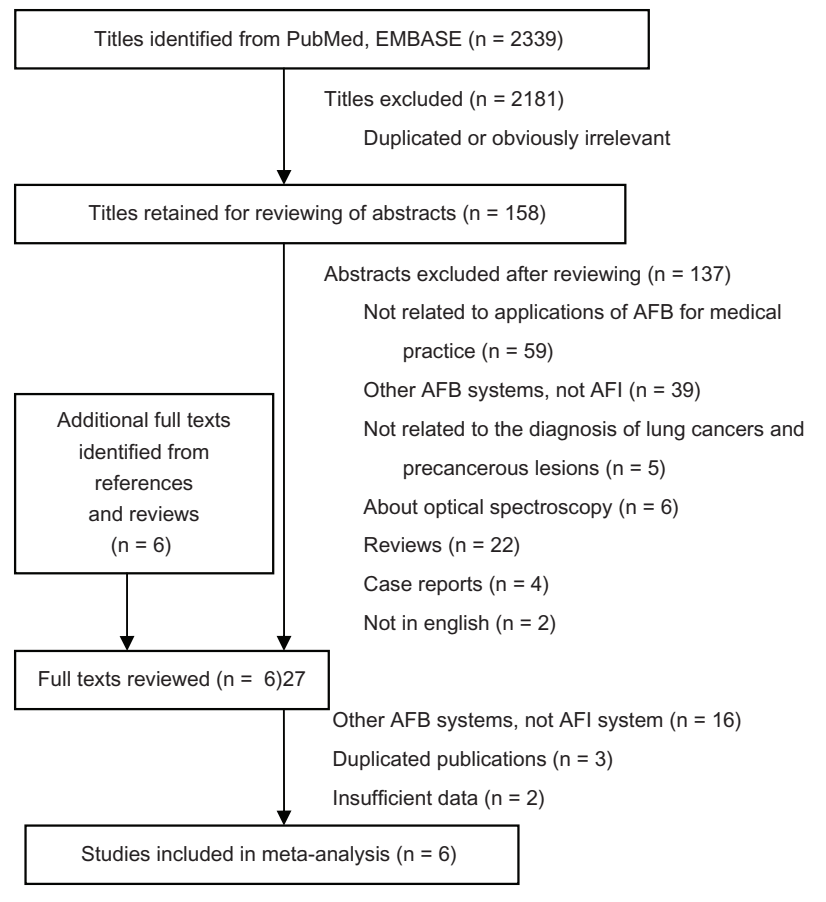

Figure 2 Summary of literature and study selection.

Abbreviations: AFB, autofluorescence bronchoscopy; AFI, autofluorescence imaging bronchoscopy; n,number.

with both systems. The mean age of the patients ranged from 56 to 69 years across the studies.

\section{Publication bias}

Linear regression testing of funnel plot asymmetry (Figure 3 and Table 3) showed that the funnel plot was asymmetric, indicating a certain degree of publication bias $(P=0.029)$. An asymmetric funnel plot would suggest that additional small studies may have been conducted but were not published because of unfavorable results.

\section{Indices of diagnostic accuracy}

The pooled sensitivity and specificity of AFI was 0.89 (95\% confidence interval [CI] 0.81-0.94) and $0.64(95 \%$ CI 0.37-0.84, Figure 4); the corresponding values for WLB were 0.67 (95\% CI 0.46-0.83) and 0.84 (95\% CI 0.74-0.91, Figure 5). The pooled positive and negative likelihood ratios were 2.5 (95\% CI 1.21-4.97) and 0.17 (95\% CI 0.08-0.36) for AFI; the corresponding values for WLB were 4.3 (95\% CI $2.13-8.52)$ and 0.39 (95\% CI $0.21-0.73)$. The pooled positive likelihood ratio for both AFI and WLB was not higher than 10 and the pooled negative likelihood ratio for both AFI and WLB was not lower than 0.1. The diagnostic odds ratio and AUC represent the overall diagnostic performance of a test. In our meta-analysis, the diagnostic odds ratio for AFI and WLB was 14.5 (95\% CI 3.76-55.63) and 10.9 (95\% CI $3.12-38.21$ ), respectively, and the AUC of AFI (Figure 6A) 
Table I quality of studies included using the quality assessment of diagnostic accuracy studies tool

\begin{tabular}{|c|c|c|c|c|c|c|}
\hline & $\begin{array}{l}\text { Chiyo } \\
\text { et al }{ }^{14}\end{array}$ & $\begin{array}{l}\text { Ueno } \\
\text { et } \mathrm{al}^{18}\end{array}$ & $\begin{array}{l}\text { Li } \\
\text { et } \mathbf{a l}^{15}\end{array}$ & $\begin{array}{l}\text { Herth } \\
\text { et al }{ }^{16}\end{array}$ & $\begin{array}{l}\text { Cetti } \\
\text { et al }{ }^{19}\end{array}$ & $\begin{array}{l}\text { Zaric } \\
\text { et al }{ }^{17}\end{array}$ \\
\hline $\begin{array}{l}\text { Spectrum of patients representative of the patients who will receive } \\
\text { the test in practice? }\end{array}$ & Yes & Yes & Yes & Yes & Yes & Yes \\
\hline Were selection criteria clearly described? & Unclear & Unclear & Unclear & Yes & Yes & Yes \\
\hline Is the reference standard likely to classify the target condition correctly? & Yes & Yes & Yes & Yes & Yes & Yes \\
\hline $\begin{array}{l}\text { Is the time period between reference standard and index test short enough } \\
\text { to prevent change of the target condition between the two tests? }\end{array}$ & Yes & Yes & Yes & Yes & Yes & Yes \\
\hline Did all subjects receive verification using a reference standard of diagnosis & Yes & Yes & Yes & Yes & Yes & Yes \\
\hline $\begin{array}{l}\text { Did all subjects receive the same reference standard regardless of the } \\
\text { index test result? }\end{array}$ & Yes & Yes & Yes & Yes & Yes & Yes \\
\hline Was the reference standard independent of the index test? & Yes & Yes & Yes & Yes & Yes & Yes \\
\hline Execution of the index test described in sufficient detail to permit replication? & Yes & Yes & Yes & Yes & Yes & Yes \\
\hline $\begin{array}{l}\text { Execution of the reference standard described in sufficient detail } \\
\text { to permit replication? }\end{array}$ & Yes & Yes & Yes & Yes & Yes & Yes \\
\hline $\begin{array}{l}\text { Were the index test results interpreted without knowledge of the results } \\
\text { of the reference standard? }\end{array}$ & Yes & Yes & Yes & Yes & Yes & Yes \\
\hline $\begin{array}{l}\text { Were the reference standard results interpreted without knowledge } \\
\text { of the results of the index test? }\end{array}$ & Yes & Yes & Yes & Yes & Yes & Yes \\
\hline $\begin{array}{l}\text { Were the same clinical data available when test results were interpreted } \\
\text { as would be available when the test is used in practice? }\end{array}$ & Yes & Yes & Yes & Yes & Yes & Yes \\
\hline Were uninterpretable/intermediate test results reported? & Unclear & Unclear & Unclear & Unclear & Unclear & Unclear \\
\hline Were withdrawals from the study explained? & Yes & Yes & Yes & Yes & Yes & Yes \\
\hline
\end{tabular}

and WLB (Figure 6B) was 0.89 (95\% CI 0.86-0.92) and 0.85 (95\% CI 0.81-0.88), respectively, indicating slightly better diagnostic performance for AFI.

\section{Evaluation of heterogeneity}

For AFI, the studies had substantial heterogeneity, with $I^{2}$ values of $81.8 \%$ and $97.4 \%$ for sensitivity and specificity, respectively (Figure 4); there was also substantial heterogeneity for WLB, with $I^{2}$ values of $87.1 \%$ and $83.9 \%$ for sensitivity and specificity, respectively (Figure 5).

\section{Discussion}

Survival rates in patients with lung cancer are strongly associated with stage of disease at the time of diagnosis. ${ }^{32}$ The five-year survival rate for patients with stage IA disease is about $73 \%$; however, for those with stage II-IV, it ranges from $46 \%$ down to as low as $9 \%$. Currently, only $16 \%$ of lung cancers are diagnosed when the disease is localized, and fewer at stage 0 , resulting in a general five-year survival rate of only about $15 \% .{ }^{33}$ As a newly developed technology, autofluorescence bronchoscopy shines some light, albeit not strongly, on this problem. It allows rapid scanning of large areas of the bronchial surface for subtle abnormalities that are not visible on WLB. ${ }^{34}$ However, in comparison with conventional WLB, some autofluorescence bronchoscopy systems have increased sensitivity but significantly reduced specificity. ${ }^{8-13}$ As an example, in a meta-analysis of autofluorescence bronchoscopy systems, Sun et $\mathrm{al}^{35}$ compared the accuracy of WLB \pm autofluorescence bronchoscopy in the diagnosis of lung cancers. It should be noted that the AFI system was not included in these autofluorescence bronchoscopy systems. Twenty-one studies were collected

Table 2 Features of studies included in meta-analysis

\begin{tabular}{|c|c|c|c|c|c|c|c|c|c|c|c|c|c|}
\hline \multirow[t]{2}{*}{ Reference } & \multirow{2}{*}{$\begin{array}{l}\text { Mean } \\
\text { age }\end{array}$} & \multirow{2}{*}{$\begin{array}{l}\text { Patients } \\
\text { (n) }\end{array}$} & \multirow{2}{*}{$\begin{array}{l}\text { Biopsies } \\
\text { (n) }\end{array}$} & \multirow[t]{2}{*}{ Control } & \multirow{2}{*}{$\begin{array}{l}\text { Positive } \\
\text { histologic } \\
\text { standard }\end{array}$} & \multicolumn{4}{|c|}{ WLB } & \multicolumn{4}{|c|}{ AFB } \\
\hline & & & & & & TP & $\mathbf{F P}$ & $\mathbf{F N}$ & TN & TP & FP & $\mathbf{F N}$ & TN \\
\hline Chiyo et $\mathrm{al}^{14}$ & 66.6 & 32 & 62 & No & $\mathrm{MI}$ & 18 & 15 & 14 & 15 & 26 & 5 & 6 & 25 \\
\hline Ueno et al ${ }^{18}$ & 69 & 31 & 64 & Yes & SEV & 14 & 4 & 5 & $4 I$ & 18 & 13 & I & 32 \\
\hline Li et $\mathrm{al}^{15}$ & 61.5 & 136 & $24 I$ & Yes & SEV & 50 & 27 & 26 & 138 & 72 & 71 & 4 & 94 \\
\hline Herth et al ${ }^{16}$ & 56.2 & 57 & 98 & No & MOC & 3 & 5 & 14 & 35 & 11 & 24 & 6 & 16 \\
\hline Cetti et al ${ }^{19}$ & ND & 49 & 81 & Yes & MO & 15 & 5 & 1 & 60 & 15 & 53 & I & 11 \\
\hline Zaric et al ${ }^{17}$ & 56 & 104 & 624 & Yes & $\mathrm{Ca}$ & 242 & 46 & 70 & 266 & 286 & 23 & 26 & 289 \\
\hline
\end{tabular}

Abbreviations: TP, true positive; FP, false positive; FN, false negative; TN, true negative; ND, not described; MI, mild dysplasia or worse; MO, moderate dysplasia or worse; $\mathrm{SEV}$, severe dysplasia or worse; MOC, moderate to severe dysplasia or carcinoma in situ; Ca, cancer; AFB, autofluorescence bronchoscopy; WLB, white light bronchoscopy. 


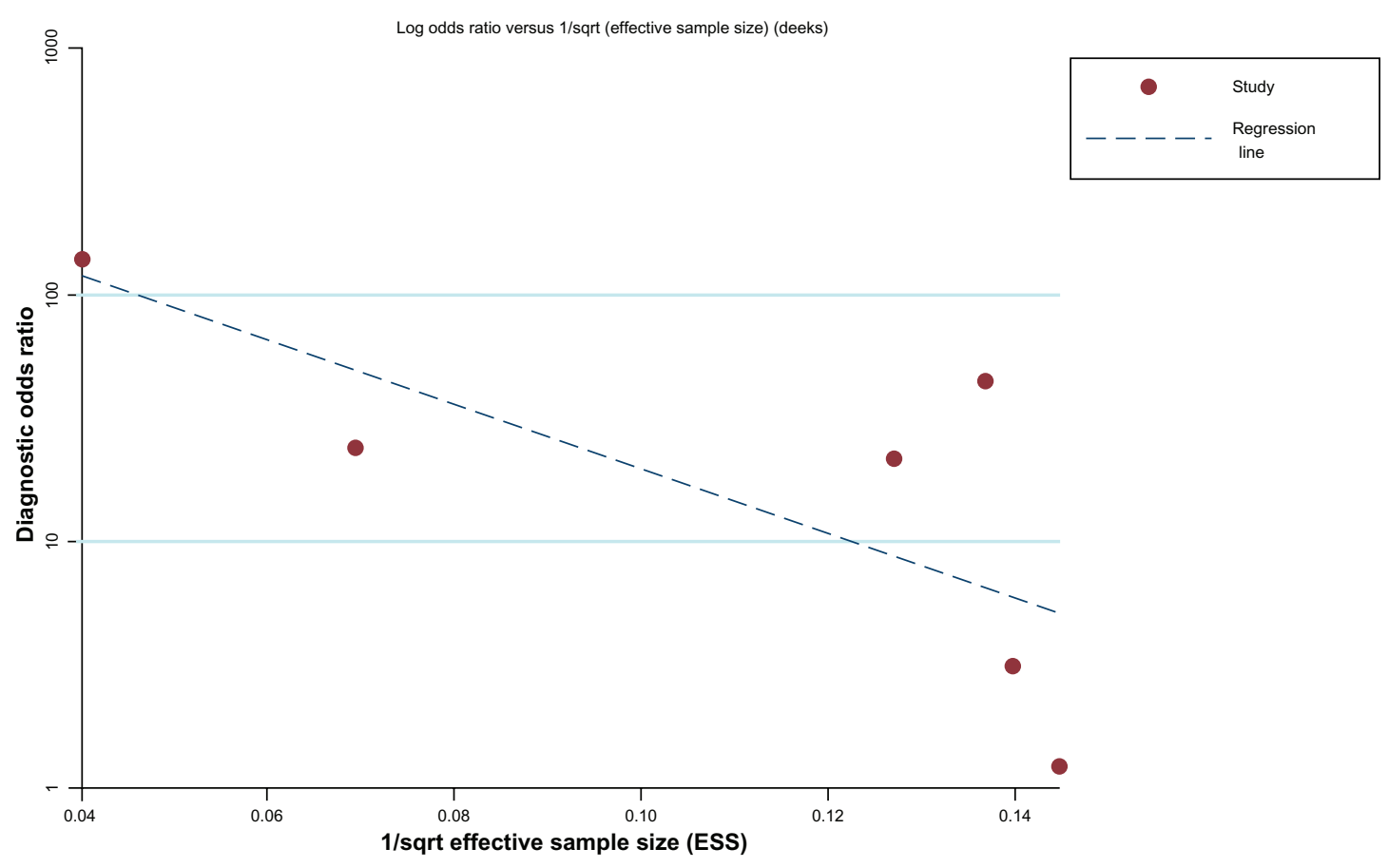

Figure 3 Linear regression test of funnel plot asymmetry.

for final analysis. The pooled relative sensitivity of WLB \pm autofluorescence bronchoscopy in detecting intraepithelial neoplasia and invasive cancer was 2.04 and 1.15 . The pooled relative specificity of WLB \pm autofluorescence bronchoscopy was as low as 0.65 . WLB combined with autofluorescence bronchoscopy seems to improve the sensitivity for detection of intraepithelial neoplasia significantly. However, this advantage over WLB alone seems much less in detecting invasive lung cancers. For example, Chen et $\mathrm{al}^{22}$ performed a meta-analysis and also concluded that autofluorescence bronchoscopy had significantly higher sensitivity and lower specificity in comparison with WLB. However, only two of the 15 studies included in their study actually included the AFI system. Including AFI with WLB when comparing autofluorescence bronchoscopy systems as a whole seems reasonable for obtaining relative sensitivity, because higher sensitivity is a characteristic common to all autofluorescence bronchoscopy systems, including AFI, LIFE, D-Light, and SAFE. However, taking the relative specificity of autofluorescence bronchoscopy systems as a whole is not appropriate.
Some reports ${ }^{14,17}$ have demonstrated that AFI may have higher specificity in comparison with other types of autofluorescence bronchoscopy. AFI displays a light-green image for normal epithelium, blue to green-blue for bleeding and inflammation, and magenta for dysplasia or cancerous lesions. This ability to distinguish dysplasia and preinvasive malignant lesions from bronchitis, hyperplasia, bleeding, and other benign lesions appears to represent a great advantage in technology which may make the specificity of AFI better. However, generally speaking, the specificity of AFI for detecting lung cancers and preneoplastic lesions remains controversial. Some reports ${ }^{14,17}$ have demonstrated that AFI has superior specificity compared with WLB, while others ${ }^{15,16}$ have shown lower specificity. It was for this reason that we felt it necessary to undertake the present meta-analysis comparing the sensitivity, specificity, and overall diagnostic performance of AFI and WLB in the diagnosis of lung cancers and precancerous lesions.

In the present meta-analysis of six studies, the pooled sensitivity of AFI (0.89) was significantly superior to that of WLB (0.67), while the pooled specificity of AFI (0.64)

Table 3 Statistical results for small study effects or publication bias

\begin{tabular}{|c|c|c|c|c|c|c|c|c|}
\hline \multirow[t]{2}{*}{ Parameter } & \multicolumn{4}{|l|}{ AFI } & \multicolumn{4}{|l|}{ WLB } \\
\hline & Coefficient & SE & $t$ & $P>|t|$ & Coefficient & SE & $t$ & $P>|t|$ \\
\hline Bias & -30.0754 & 9.0667 & -3.32 & 0.029 & -7.3928 & 12.7558 & -0.58 & 0.593 \\
\hline Intercept & 5.9833 & 0.6866 & 8.71 & 0.001 & 3.1850 & 0.9658 & 3.30 & 0.030 \\
\hline
\end{tabular}

Abbreviations: AFI, autofluorescence imaging bronchoscopy; WLB, white light bronchoscopy; SE, standard error of the mean. 

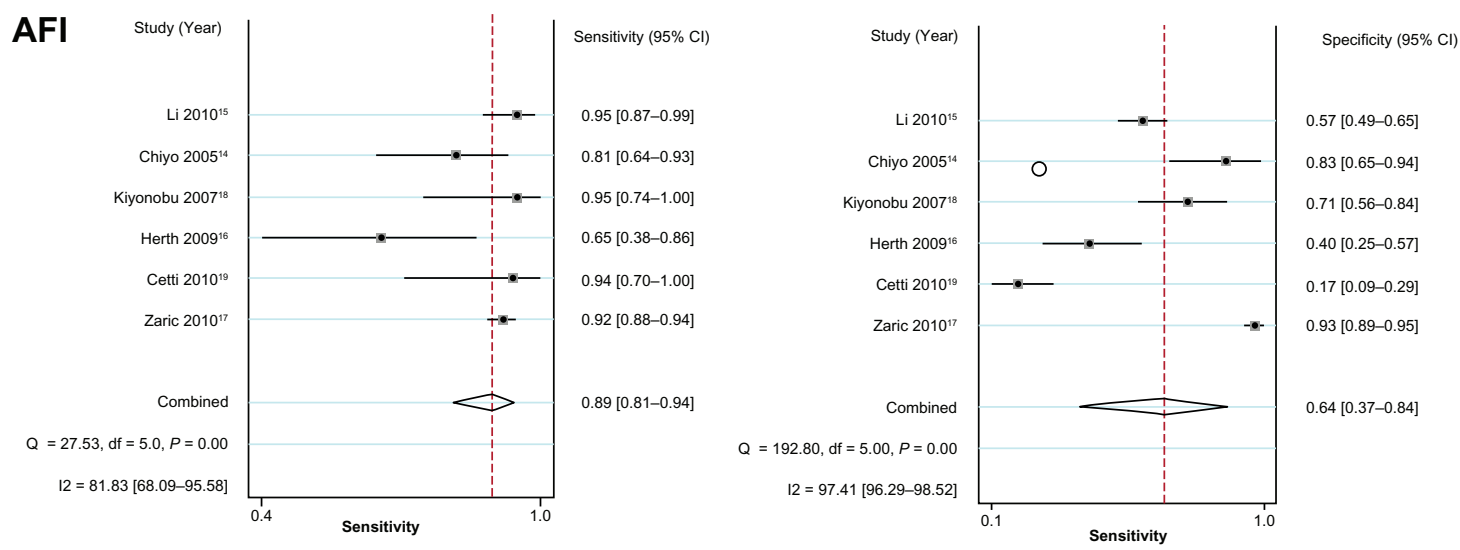

Figure 4 Forest plot of sensitivity and specificity of AFI for detecting lung cancers and precancerous lesions.

Abbreviation: AFI, autofluorescence imaging bronchoscopy; $\mathrm{Cl}$, confidence interval; df, degree of freedom; Q, cochran's heterogeneity statistic.

was lower than that of WLB (0.84). The diagnostic odds ratio for AFI and WLB was 14.5 and 10.9 and the AUC of AFI and WLB was 0.89 and 0.85 , respectively. The diagnostic odds ratio and AUC represent the overall diagnostic performance of the studies included. Therefore, the pooled results demonstrate that the sensitivity of AFI is higher than for WLB while the specificity of AFI is lower, and indicate slightly better overall diagnostic performance for AFI. The pooled positive likelihood ratio for both AFI and WLB was not higher than 10, and the pooled negative likelihood ratio for both AFI and WLB was not lower than 0.1. For both AFI and WLB, a positive endoscopic finding cannot make a definite diagnosis of lung cancer or a precancerous lesion, while a negative endoscopic finding cannot exclude lung cancer or a precancerous lesion.

The quality of WLB images has improved with the advent of video bronchoscopy, but is still plagued by limitations in diagnosing early-stage mucosal lesions. More specifically, because WLB is ineffective for detecting lesions smaller than $5 \mathrm{~mm}$ in diameter, the surface mucosa typically appears relatively normal on WLB when earlystage mucosal lesions are only a few cell layers thick. AFI is a new technology exploiting the autofluorescent nature of the bronchial mucosa to detect tiny and superficial lesions. The AFI can identify abnormal mucosa in different colors. Therefore, compared with WLB, AFI may be better for identifying early-stage mucosal lesions, thereby improving the diagnostic sensitivity of bronchoscopy for early-stage cancer and precancerous lesions. ${ }^{24,36}$ The increased sensitivity of AFI bronchoscopy can further improve the ability of trainees to make a correct diagnosis, and suggests an important role for AFI in the training of bronchoscopists at teaching hospitals. ${ }^{19}$

Although AFI can detect early-stage lung cancers and precancerous lesions on the bronchial surface mucosa in the central airways with quite high sensitivity, its relatively low specificity may be a problem. Increased blood content or vessel growth and increased thickening of the bronchial
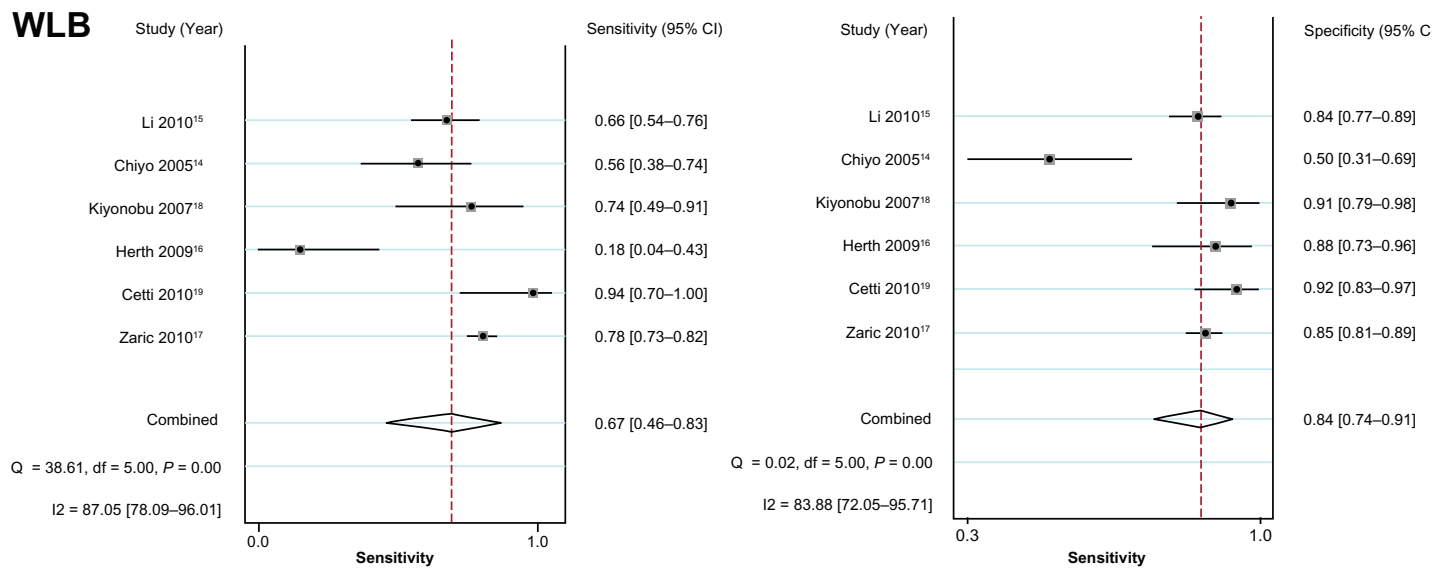

Figure 5 Forest plot of sensitivity and specificity of WLB for detecting lung cancers and precancerous lesions.

Abbreviation: WLB, white light bronchoscopy; $\mathrm{Cl}$, confidence interval; df, degree of freedom; $\mathrm{Q}$, Cochran's heterogeneity statistic. 


\section{A SROC with confidence and predictive ellipses}

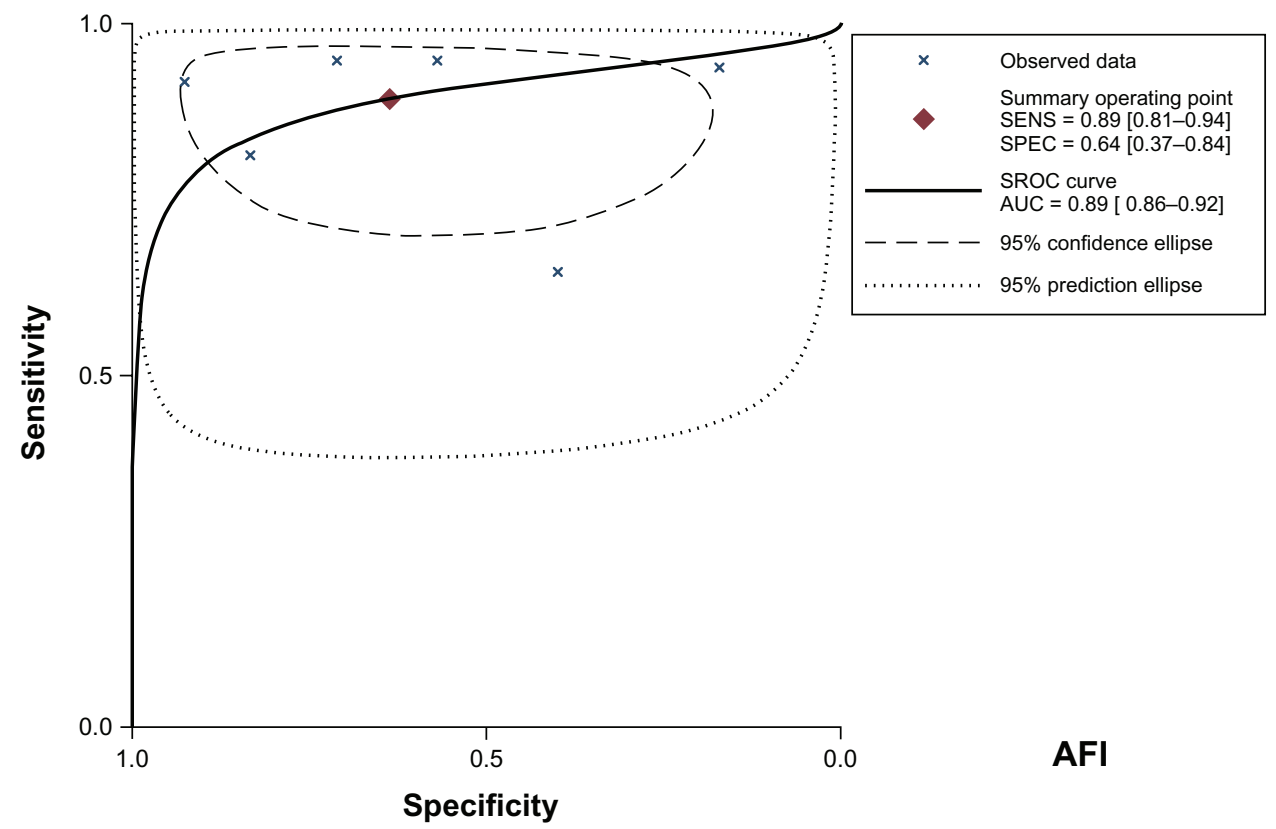

B SROC with confidence and predictive ellipses
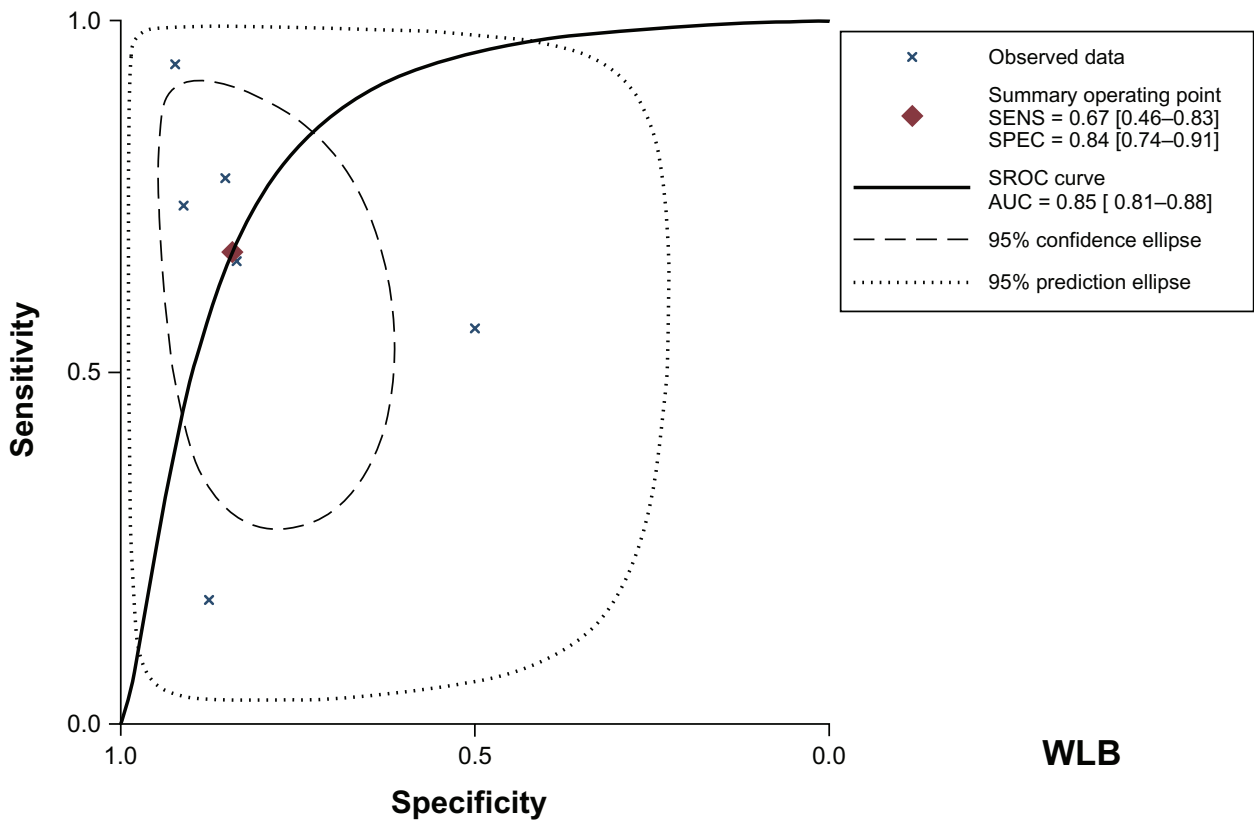

Figure 6 Summary receiver operating characteristic curves for (A) AFI and (B) WLB.

Abbreviations: AFI, autofluorescence imaging bronchoscopy; WLB, white light bronchoscopy; SROC, summary receiver operating characteristic; SENS, sensitivity; SPEC, specificity; AUC, area under the curve.

epithelium are thought to be involved in areas of abnormal autofluorescence irrespective of malignancy. Increased blood content and thickening of the epithelium also occurs in other chronic inflammatory bronchial lesions arising from chronic bronchitis, bronchial asthma, or other benign morbidities, so incorrect diagnoses may be made when these "abnormal" AFI images are detected, and may account for why the false positive rate for AFI is high and the specificity is relatively lower than for WLB. Another explanation is that lesions identified under autofluorescence bronchoscopy and confirmed as false positives may carry molecular genetic lesions associated with malignancy, despite a normal histological appearance. ${ }^{37,38}$ 
The relative lower specificity of AFI may lead to more biopsies being taken, at greater cost to the health care system. However, the pattern of high sensitivity and low specificity of AFI is similar to that of some other imaging modalities, such as CT, which is generally used in the diagnosis of small malignant nodules, or other cancer screening tools, such as mammography and occult blood testing. ${ }^{39}$

In our opinion, the combined use of AFI and WLB may be superior to WLB alone, and could result in better diagnostic yields with higher sensitivity and without significantly compromising specificity. ${ }^{40}$ In clinical practice, AFI and WLB are incorporated into one endoscopic system, making it convenient to switch between these two modes of examination by just pressing a button. Systematic training in AFI may be necessary for endoscopists because not only is subjective assessment of AFI images needed but also objective color tone analysis is important for distinguishing lung cancers and precancerous lesions from bronchitis to improve specificity. ${ }^{14}$

Although heterogeneity among studies of diagnostic accuracy is an inevitable problem for meta-analysis, ${ }^{26}$ we discuss here the possible sources of heterogeneity as limitations of this review.

\section{Inclusion criteria}

Chiyo et $\mathrm{al}^{14}$ and Ueno et $\mathrm{al}^{18}$ included patients with suspected or known lung cancers in their studies of the efficacy of AFI in the diagnosis of cancers and precancerous lesions. Li et a ${ }^{15}$ did a prospective clinical study assessing the clinical value of AFI in an examination of the airways and recruited patients who had undergone both AFI and WLB for general diagnosis and for postoperative follow-up. Herth et $\mathrm{al}^{16}$ compared the yields of AFI and WLB in the diagnosis of intraepithelial neoplasia, and included patients who were at high risk for lung cancer but had no clinical signs raising suspicion for central airway malignancy. A study by Cetti et $\mathrm{al}^{19}$ evaluated the diagnostic performance of AFI in patients with suspected lung cancer, and excluded those with an established diagnosis of lung cancer involving the central airways. A study by Zaric et $\mathrm{al}^{17}$ evaluated the performance of AFI in the assessment of tumor extent (margins) and recruited patients with suspected lung cancer but excluded those with suspected or known lung cancer metastases. Although these authors recruited patients for different purposes using different inclusion criteria, the common key point of these studies is that they compared the sensitivity and specificity of AFI and WLB in detecting lung cancers and precancerous lesions, so we believe that it was acceptable to collect them together for a meta-analysis.

\section{Endoscopic classification and positive histological standard}

In study by Chiyo et al, ${ }^{14}$ a magenta image was classified as positive for malignant or preinvasive lesions, whereas biopsies with a histological grading of dysplasia or worse were considered positive. Ueno et $\mathrm{al}^{18}$ evaluated their AFI findings using a four-point scale and evaluated WLB finding using a threepoint scale. AFI-III (images appeared magenta) and WLB-III (changes suggesting severe dysplasia and cancer) were considered as positive findings endoscopically; biopsies with severe dysplasia or worse were considered as positive histologically. Li et $\mathrm{al}^{15}$ classified endoscopic lesions into three grades in AFI or WLB. For AFI, AFI-II (images appeared pink or brown) and AFI-III (images appeared classic magenta) were considered as abnormal findings and for WLB, WLB-II (including hyperemia, edema, thickness, color changes, and regression or buckling of mucosal vessels) and WLB-III (including granulation of the bronchial mucosa or visible neoplasm) were considered as abnormal findings. In this study, biopsies with a histological grading of severe dysplasia or worse were considered positive. Herth et a ${ }^{16}$ evaluated AFI and WLB findings using a fourpoint scale, ie, normal, abnormal but not suspicious, suspicious for intraepithelial neoplasia, or tumor. Any lesion that met the criteria of "suspicious for intraepithelial malignancy" was classified as a positive finding on bronchoscopy for preinvasive neoplasia; biopsies with a histological grading of moderate to severe dysplasia or carcinoma in situ were considered positive for intraepithelial neoplasia. Cetti et $\mathrm{al}^{19}$ graded the appearance of the mucosa on bronchoscopy as normal, nonspecific abnormality or inflammation suspicious for cancer, or a definite tumor. Lesions meeting the criteria of "suspicious of cancer" or "definite tumor" were classified as a positive finding on bronchoscopy, and biopsies with a histological grading of moderate dysplasia or worse were considered positive. In the study by Zaric et al, ${ }^{17}$ red-brownish or magenta-colored areas were defined as pathologic areas by AFI, and biopsies with a histologic grading of carcinoma were considered as positive. As the authors of a meta-analysis, we accepted the endoscopic classification and positive histological standard made by the authors of the original articles because they had their own appropriate considerations regarding research purposes and were best placed to make decisions when choosing their positive criteria.

\section{Biopsy strategy}

In five of the six studies ${ }^{14-16,18,19}$ included in our meta-analysis, endobronchial biopsies were taken from the cores of areas 
that appeared suspicious under either AFI or WLB mode. The remaining study ${ }^{17}$ evaluated the value of AFI versus WLB in assessing tumor extension in the central airways for surgical decision-making, and biopsies were then taken from the margins of visualized pathologically changed mucosa. However, the key objective of all these studies was still to compare the sensitivity and specificity of AFI and WLB in detecting lung cancers and precancerous lesions.

\section{Conclusion}

Our meta-analysis indicates the sensitivity of AFI is higher than that of WLB and that the specificity of AFI is lower than that of WLB in detecting lung cancers or precancerous lesions. The overall diagnostic performance of AFI is slightly better than that of WLB. Combined use of AFI and WLB is recommended and may result in better diagnostic yields with higher sensitivity and without compromising specificity to a significant extent. ${ }^{40}$ The additional sensitivity provided by AFI may improve the ability of trainees to make a correct diagnosis during training ${ }^{19}$ and the false positive rate of AFI may be decreased by objective color tone analysis. ${ }^{14}$ For all these reasons, AFI should find its place in routine bronchoscopic examination and may improve the outcome of endoscopic diagnosis of lung cancers and precancerous lesions.

\section{Acknowledgment}

This study was supported by grants from the National Natural Science Foundation of China (81270144, 30800507, $81170071)$. We are grateful for the information and corrections made by Danielle Speer and Ambrose Chiang in the Division of Pulmonary and Critical Care Medicine at Duke University Medical Center, Durham, NC, USA.

\section{Disclosure}

None of the authors has a financial relationship with any commercial entity having an interest in the subject of this work and therefore, report no other conflicts of interest in this work.

\section{References}

1. Greenlee RT, Murray T, Bolden S, Wingo PA. Cancer statistics, 2000. CA Cancer J Clin. 2000;50(1):7-33.

2. Piérard P, Faber J, Hutsebaut J, et al. Synchronous lesions detected by autofluorescence bronchoscopy in patients with high-grade preinvasive lesions and occult invasive squamous cell carcinoma of the proximal airways. Lung Cancer. 2004;46(3):341-347.

3. Sutedja TG, Codrington H, Risse EK, et al. Autofluorescence bronchoscopy improves staging of radiographically occult lung cancer and has an impact on therapeutic strategy. Chest. 2001;120(4): $1327-1332$

4. Woolner LB, Fontana RS, Cortese DA, et al. Roentgenographically occult lung cancer: pathologic findings and frequency of multicentricity during a 10-year period. Mayo Clin Proc. 1984;59(7):453-466.
5. Lycette RM, Leslie RB. Fluorescence of malignant tissue. Lancet. 1965;2(7409):436.

6. Lam S, MacAulay C, leRiche JC, Palcic B. Detection and localization of early lung cancer by fluorescence bronchoscopy. Cancer. 2000; 89(Suppl 11):2468-2473.

7. Kakihana M, Il KK, Okunaka T, et al. Early detection of bronchial lesions using system of autofluorescence endoscopy (SAFE) 1000. Diagn Ther Endosc. 1999;5(2):99-104.

8. Edell E, Lam S, Pass H, et al. Detection and localization of intraepithelial neoplasia and invasive carcinoma using fluorescence-reflectance bronchoscopy: an international, multicenter clinical trial. $J$ Thorac Oncol. 2009;4(1):49-54.

9. Jang TW, Oak CH, Chun BK, Jung MH. Detection of pre-invasive endobronchial tumors with D-light/autofluorescence system. J Korean Med Sci. 2006;21(2):242-246.

10. Venmans BJ, Van Boxem TJ, Smit EF, Postmus PE, Sutedja TG. Results of two years expenience with fluorescence bronchoscopy in detection of preinvasive bronchial neoplasia. Diagn Ther Endosc. 1999;5(2):77-84.

11. Kusunoki Y, Imamura F, Uda H, Mano M, Horai T. Early detection of lung cancer with laser-induced fluorescence endoscopy and spectrofluorometry. Chest. 2000;118(6):1776-1782.

12. Hirsch FR, Prindiville SA, MillerYE, et al. Fluorescence versus whitelight bronchoscopy for detection of preneoplastic lesions: a randomized study. J Natl Cancer Inst. 2001;93(18):1385-1391.

13. Hirsch FR, Franklin WA, Gazdar AF, Bunn PA Jr. Early detection of lung cancer: clinical perspectives of recent advances in biology and radiology. Clin Cancer Res. 2001;7(1):5-22.

14. Chiyo M, Shibuya K, Hoshino H, et al. Effective detection of bronchial preinvasive lesions by a new autofluorescence imaging bronchovideoscope system. Lung Cancer. 2005;48(3):307-313.

15. Li Y, Li X, Sui XZ, et al. Comparison of the autofluorescence bronchoscope and the white light bronchoscope in airway examination. Chin J Cancer. 2010;29(12):1018-1022.

16. Herth FJ, Eberhardt R, Anantham D, Gompelmann D, Zakaria MW, Ernst A. Narrow-band imaging bronchoscopy increases the specificity of bronchoscopic early lung cancer detection. J Thorac Oncol. 2009;4(9): 1060-1065.

17. Zaric B, Becker HD, Perin B, et al. Autofluorescence imaging videobronchoscopy improves assessment of tumor margins and affects therapeutic strategy in central lung cancer. Jpn J Clin Oncol. 2010;40(2): 139-145.

18. Ueno K, Kusunoki Y, Imamura F, et al. Clinical experience with autofluorescence imaging system in patients with lung cancers and precancerous lesions. Respiration. 2007;74(3):304-308.

19. Cetti EJ, Nicholson AG, Singh S, Wells AU, Shah PL. An evaluation of a videobronchoscopy-based autofluorescence system in lung cancer. Eur Respir J. 2010;35(5):1185-1187.

20. Zaric B, Perin B, Becker HD, et al. Autofluorescence imaging videobronchoscopy in the detection of lung cancer: from research tool to everyday procedure. Expert Rev Med Devices. 2011;8(2):167-172.

21. Keith RL, MillerYE, Gemmill RM, et al. Angiogenic squamous dysplasia in bronchi of individuals at high risk for lung cancer. Clin Cancer Res. 2000;6(5):1616-1625.

22. Chen W, Gao X, Tian Q, Chen L. A comparison of autofluorescence bronchoscopy and white light bronchoscopy in detection of lung cancer and preneoplastic lesions: a meta-analysis. Lung Cancer. 2011;73(2): 183-188.

23. Palcic B, Lam S, Hung J, MacAulay C. Detection and localization of early lung cancer by imaging techniques. Chest. 1991;99(3):742-743.

24. Lam S, MacAulay C, Hung J, LeRiche J, Profio AE, Palcic B. Detection of dysplasia and carcinoma in situ with a lung imaging fluorescence endoscope device. J Thorac Cardiovasc Surg. 1993;105(6): 1035-1040.

25. Whiting P, Rutjes AW, Reitsma JB, Bossuyt PM, Kleijnen J. The development of QUADAS: a tool for the quality assessment of studies of diagnostic accuracy included in systematic reviews. BMC Med Res Methodol. 2003;3:25. 
26. Harbord RM, Deeks JJ, Egger M, Whiting P, Sterne JA. A unification of models for meta-analysis of diagnostic accuracy studies. Biostatistics. 2007;8(2):239-251.

27. Higgins JP, Thompson SG, Deeks JJ, Altman DG. Measuring inconsistency in meta-analyses. BMJ. 2003;327(7414):557-560.

28. Glas AS, Lijmer JG, Prins MH, Bonsel GJ, Bossuyt PM. The diagnostic odds ratio: a single indicator of test performance. $J$ Clin Epidemiol. 2003;56(11):1129-1135.

29. Sousa MR, Ribeiro AL. Systematic review and meta-analysis of diagnostic and prognostic studies: a tutorial. Arq Bras Cardiol. 2009;92(3):229-238.

30. Martin JL, Williams KS, Abrams KR, et al. Systematic review and evaluation of methods of assessing urinary incontinence. Health Technol Assess. 2006;10(6):1-132.

31. Moses LE, Shapiro D, Littenberg B. Combining independent studies of a diagnostic test into a summary ROC curve: data-analytic approaches and some additional considerations. Stat Med. 1993;12(14): 1293-1316.

32. Motta G, Carbone E, Spinelli E, Nahum MA, Testa T, Flocchini GP. Considerations about tumor size as a factor of prognosis in NSCLC. Ann Ital Chir. 1999;70(6):893-897.

33. Goldstraw P, Crowley J, Chansky K, et al. The IASLC Lung Cancer Staging Project: proposals for the revision of the TNM stage groupings in the forthcoming (seventh) edition of the TNM Classification of malignant tumours. J Thorac Oncol. 2007;2(8):706-714.
34. Ohtani K, Lee AM, Lam S. Frontiers in bronchoscopic imaging Respirology. 2012;17(2):261-269.

35. Sun J, Garfield DH, Lam B, et al. The value of autofluorescence bronchoscopy combined with white light bronchoscopy compared with white light alone in the diagnosis of intraepithelial neoplasia and invasive lung cancer: a meta-analysis. J Thorac Oncol. 2011;6(8):1336-1344.

36. Lam S, Macaulay C, Leriche JC, Ikeda N, Palcic B. Early localization of bronchogenic carcinoma. Diagn Ther Endosc. 1994;1(2):75-78.

37. Wistuba II, Lam S, Behrens C, et al. Molecular damage in the bronchial epithelium of current and former smokers. J Natl Cancer Inst. 1997; 89(18):1366-1373.

38. Helfritzsch H, Junker K, Bartel M, Scheele J. Differentiation of positive autofluorescence bronchoscopy findings by comparative genomic hybridization. Oncol Rep. 2002;9(4):697-701.

39. Henschke CI, McCauley DI, Yankelevitz DF, et al. Early Lung Cancer Action Project: overall design and findings from baseline screening. Lancet. 1999;354(9173):99-105.

40. Häussinger K, Becker H, Stanzel F, et al. Autofluorescence bronchoscopy with white light bronchoscopy compared with white light bronchoscopy alone for the detection of precancerous lesions: a European randomised controlled multicentre trial. Thorax. 2005;60(6):496-503.
Patient Preference and Adherence

\section{Publish your work in this journal}

Patient Preference and Adherence is an international, peer-reviewed, open access journal focusing on the growing importance of patient preference and adherence throughout the therapeutic continuum. Patient satisfaction, acceptability, quality of life, compliance, persistence and their role in developing new therapeutic modalities and compounds to

\section{Dovepress}

optimize clinical outcomes for existing disease states are major areas of interest. This journal has been accepted for indexing on PubMed Central. The manuscript management system is completely online and includes a very quick and fair peer-review system. Visit http://www.dovepress.com/ testimonials.php to read real quotes from published authors. 